\title{
Effectiveness of automated appointment reminders in psychosis community services: a randomised controlled trial $\dagger$
}

Eugenia Kravariti* , Christopher Reeve-Mates*, Rafaela Da Gama Pires*, Elias Tsakanikos*, Daniel Hayes, Siobhan Renshaw, Sarah McAllister, Vishal Bhavsar, Pam Patterson, Emily Daley, Jane Stewart, Megan Pritchard, Hitesh Shetty, Rosalind Ramsay, Rocio Perez-Iglesias* and Philip McGuire*

\section{Summary}

We report on the first open-label, parallel group randomised controlled trial of automated appointment reminders in a psychosis community service in the UK. Ninety-five patients were randomly allocated to receiving/not receiving automated messaging reminders 7 days and 1 day before appointments. All 'Attended' and 'Missed' appointment outcomes over 6 months were analysed using cluster regression analysis. Reminded appointments were significantly more frequently attended than non-reminded appointments (unadjusted odds ratio $(\mathrm{OR})=3.54$, $95 \% \mathrm{Cl} 1.36-9.22, P=0.01$; adjusted $\mathrm{OR}=2.95,95 \% \mathrm{Cl} 1.05-8.85$, $P<0.05)$. Automated messaging reminders can provide a robust strategy for promoting engagement with psychosis services.

\section{Declaration of interest}

The authors have no competing financial interests to declare in relation to the current work. Sarah McAllister was supported by a King’s Undergraduate Research Fellowship.

\section{Copyright and usage}

(c) The Royal College of Psychiatrists 2018. This is an Open Access article, distributed under the terms of the Creative Commons Attribution-NonCommerical-

NoDerivatives licence (http://creativecommons.org/licenses/ by-nc-nd/4.0/), which permits non-commercial re-use, distribution, and reproduction in any medium, provided the original work is unaltered and is properly cited. The written permission of Cambridge University Press must be obtained for commercial re-use or in order to create a derivative work
Approximately 1 in 10 out-patient hospital appointments are missed in the UK every year, costing the National Health System (NHS) an astounding $£ 745$ million in $2012 / 13$. 'No shows' are up to three times higher in mental health services, ${ }^{2,3}$ increasing risk for functional decline, symptom exacerbation and suicide. ${ }^{4,5}$

Across health services, automated appointment reminders reduce non-attendance by $29 \%,{ }^{6}$ but have been infrequently investigated in severe mental illness. ${ }^{3,7-10}$ High-quality studies that can inform the effectiveness, practicality and acceptability of this approach in the context of psychosis are scarce. ${ }^{7,8,10,11}$ We report on the first randomised controlled trial (RCT) of automated short message service (SMS) appointment reminders in a psychosis community service (PCS) in the UK. We hypothesised that patients randomly allocated to the SMS intervention would miss significantly fewer appointments compared with controls.

\section{Methods}

The study was an open-label RCT with a parallel group design (NHS Research Ethics Committee reference 13/LO/0497; ClinicalTrials.gov registration number NCT01793220; full record accessible at https://clinicaltrials.gov/). Recruitment was conducted and the primary outcome ('appointment attendance') assessed in February-July 2014 in the Psychosis Recovery and Support Community Service in Lambeth South, London. The PCS underwent radical restructuring in summer 2014. This led to relocation of patients and their clinical teams to other PCSs, and to an unplanned study termination before secondary outcomes could be

$\dagger$ The original publication of this article did not include the full affiliation of Dr Rocio Perez-Iglesias. This version has been updated to include this information and a corrigendum has been published.

* These authors contributed equally to this manuscript (joint first or joint last authors). assessed. By July 2014, 95 participants had entered the study according to the following inclusion criteria: mobile phone ownership, decisional capacity (assessed by a capacity-to-consent scale) ${ }^{12}$ willingness to receive SMS reminders and written informed consent to participate. Participants were randomly allocated to receiving/not receiving automated text message appointment reminders on their personal mobile phones 7 days and 1 day before each appointment. The intervention was an adjunct to routine reminder practices (e.g. letters) in the service.

Random allocation was balanced (ratio $1: 1$ ), leading to approximately equal group sizes (46 participants in the intervention arm, 49 participants in the control arm). D.H. generated the random allocation sequence prior to the study, using an online tool (https:// www.random.org/sequences/) which randomly divided integers into 'intervention' and 'control' columns. Allocation outcome was determined by whether each participant's serial recruitment number matched an integer in the 'intervention' or the 'control' column. Participants were recruited, enrolled and assigned to groups by C.R.-M., R.D.G.P., S.R. and S.M., who were informed of each consecutive allocation outcome by E.K.. The same authors enabled the automated reminders using the inbuilt functionality of ePJS (electronic patient journey system), the electronic clinical records system used in the PCS (where this functionality had never been enabled prior to the study). The reminder text read 'You have an appointment with Lambeth South at [TIME] on [DATE]. Please call XXXXXXXXXXX if you cannot come'. Participant appointments and attendance outcomes were separately entered into ePJS by the research and clinical teams, respectively, as soon as they were scheduled and known.

Data extraction and analysis were conducted from September 2014 to March 2015, using the Clinical Records Interactive Search (CRIS) system ${ }^{13}$ and Stata 11.0 , respectively. CRIS provides authorised researchers with regulated access to over 250000 fully anonymised ePJS records. Ethical approval for CRIS as an anonymisation portal for secondary analyses was provided by an NHS 
research ethics committee in accordance with the Declaration of Helsinki, as well as by the Institute of Psychiatry's Institutional Review Board. To enable data extraction, the research team tagged all participant appointments in ePJS at the point of appointment entry using unmodifiable study- and arm-specific tags. These were later used as search terms in CRIS to extract the data.

The unit of analysis was 'appointment'. Power analyses using an alpha of 0.05 , power of 0.80 and various simulated effects ranging from small to large determined a desired size of $88-1511$ appointments per study group (supplementary Table 1, available at https://doi.org/10.1192/bjo.2017.7).

Observations relating to appointments scheduled for the same individual (4.73 appointments on average) were dependent. Therefore, 'Attended' and 'Missed' outcomes were compared between the study arms using logistic regression analysis for clustered observations, before and after adjusting for variables that differed significantly between groups (see Results) or that significantly predicted attendance in preliminary logistic regression analyses (only 'Diagnosis'; see supplementary Table 2). We next repeated the analysis in the schizophrenia subgroup.

A CONSORT 2010 information checklist and flow diagram of the study stages are presented in supplementary Table 3 and Fig. 1, respectively.

\section{Results}

Of the 95 participants, six intervention and 14 control participants $\left(\chi^{2}=3.44 ; P=0.06\right)$ had no appointments (and therefore no identifying tags) entered in ePJS during the study period. In the context of a fully anonymised analysis, it was not possible to extract and analyse the respective clinical/demographic data. The remaining 75 participants (40 intervention, 35 control) contributed a total of 355 appointments (intervention, 197; control, 158); 113 7-day reminders and 168 1-day reminders were sent without error to mobile phones owned by participants in the intervention arm. These figures suggest that of the 197 intervention appointments, 113 received both reminders, 55 received only 1 -day reminders (because the appointment was scheduled for a date less than 7 days later) and 29 received no reminders.

The characteristics of the analytic cohort $(n=75)$ are presented in Table 1. The intervention and control groups differed significantly only in diagnosis (Table 1). As there were no significant differences in 'Cancelled', 'Not Known/Recorded' and 'Other' appointment outcomes among the study groups (Table 1), the analysis focused on appointments with the main outcomes of interest, i.e. 'Attended' $v$. 'Missed' $(n=275)$ (Table 1). Of the 151 reminded appointments with such outcomes, 138 (91\%) were 'Attended' and 13 (9\%) 'Missed', compared with 93 (75\%) and 31 (25\%), respectively, of the 124 non-reminded appointments (unadjusted odds ratio $(\mathrm{OR})=3.54,95 \% \mathrm{CI} 1.36-9.22, P=0.01)$.

After adjusting for diagnosis, the OR remained robust and statistically significant (adjusted OR $=2.95,95 \%$ CI 1.05-8.85, $P<$ $0.05)$. In the schizophrenia sub-analysis, the OR remained robust, but the statistical significance became a trend $(\mathrm{OR}=3.78,95 \% \mathrm{CI}$ $0.82-17.42, P=0.09)$, possibly reflecting the decrease in statistical power (148 appointments: 92 intervention, 56 control).

\section{Discussion}

Against a background of routine SMS implementations in the NHS, the systematic examination of their use in patients with psychosis is a novelty of the present study. This population presents unique challenges, including reduced insight, cognitive deficits, psychopathology, confidentiality concerns, and unemployment affecting rates of mobile phone ownership. ${ }^{14,15}$ Notwithstanding these barriers, the balance of attended versus missed appointments in our study was markedly improved among participants who received automated messaging reminders (91\% v. 9\%) compared with those who did not (75\% v. 25\%).

Our RCT was fully integrated into the routine practice of a PCS; addressed a pervasive problem with profound financial and health costs; and had high ecological validity, excellent confidentiality and anonymity, no known harmful effects, and important cost and clinical implications. Owing to the redistribution of patients across PCSs in summer 2014, the study was terminated before

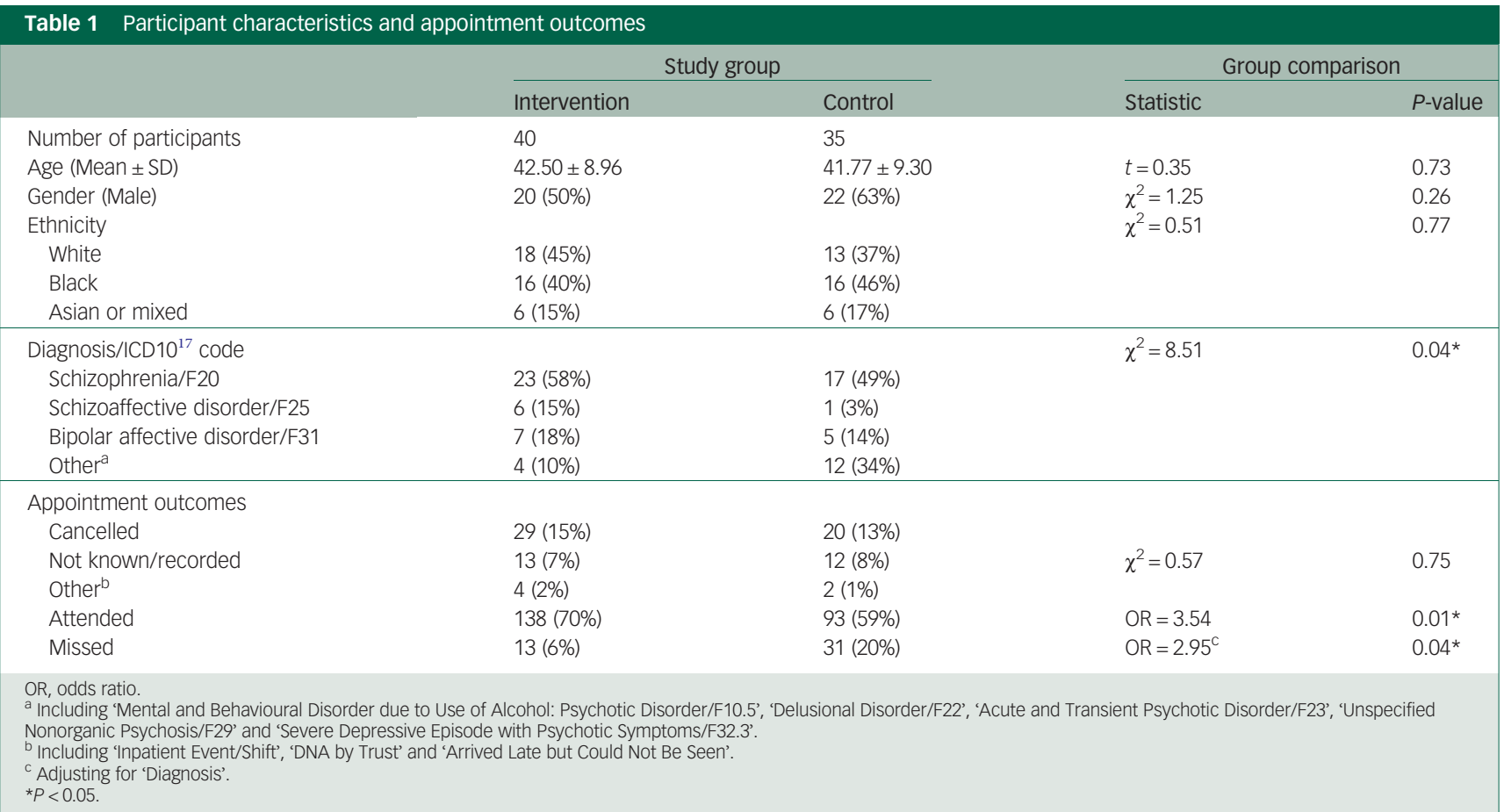


patient perceptions and other secondary study outcomes could be assessed. A notable methodological weakness is that the study would have lacked power to detect hypothetical smaller effects. Although our participants' random allocation to the study arms was balanced $(1.07: 1.00)$, the number of appointments was slightly unequal $(1.25: 1.00)$, pointing to a potential study limitation. Indeed, equal allocation is the most efficient approach in RCTs. ${ }^{16}$ Although the reminder text invited participants to notify staff of their inability to attend, the study arms showed comparable cancellation rates. As the numbers of non-reminded $(n=29)$ and 'Cancelled' $(n=29)$ appointments in the intervention arm were exactly the same, a likely explanation is that most, if not all, cancelled appointments were cancelled before any reminders were sent, thus minimising any reminder effects on cancellation rates. As the anonymous reminder data were provided directly by the South London and Maudsley (SLaM) clinical systems team rather than retrieved from CRIS, we cannot verify this highly plausible explanation. Finally, although we cannot exclude the possibility that some participants perceived the reminders as patronising and intrusive, it is encouraging that no individual complained or asked to withdraw from the study. Further, it is worth noting that a more welcoming messaging reminder might have been even more effective than our neutrally worded prompt.

Our findings add to emerging evidence ${ }^{3,7-11}$ that automated messaging reminders provide a feasible and robust strategy for engaging people with psychosis in healthcare. Although still a comparatively untapped resource for psychosis, SMS applications show exciting potential for promoting positive patient outcomes and transforming healthcare.

\footnotetext{
Eugenia Kravariti, PhD, Christopher Reeve-Mates, MSC, Rafaela Da Gama Pires, PGDip, King's College London, Department of Psychosis Studies, Institute of Psychiatry, Psychology \& Neuroscience, London, UK; Elias Tsakanikos, PhD, University of Roehampton, Department of Psychology, London, UK; Daniel Hayes, MSc, King's College London, Department of Psychosis Studies, Institute of Psychiatry, Psychology \& Neuroscience, London, UK, and Evidence Based Practice Unit, UCL and Anna Freud National Centre for Children and Families, London, UK; Siobhan Renshaw, BSC, Brune University London, Department of Psychology, Middlesex, UK; Sarah McAllister, RMN MRes, BSC Hons, Vishal Bhavsar, MD, King's College London, Department of Psychosis Studies, Institute of Psychiatry, Psychology \& Neuroscience, London, UK, and South London and Maudsley NHS Foundation Trust, Bethlem Royal Hospital, Kent, UK; Pam Patterson, RGN, RMN, Emily Daley, MRCPsych, Jane Stewart, South London Pam Patterson, RGN, RMN, Emily Daley, MRCPsych, Jane Stewart,
and Maudsley NHS Foundation Trust, Bethlem Royal Hospital, Kent, UK; Megan Pritchard, MSc, King's College London, Department of Psychological Medicine Institute of Psychiatry, Psychology \& Neuroscience, London, UK, and The National Institute for Health Research (NIHR) Biomedical Research Centre (BRC) at South London and Maudsley NHS Foundation Trust and the Institute of Psychiatry, Psychology \& Neuroscience at King's College London, King's Health Partners Academic Health Neuroscience at King's College London, King's Health Partners Academic Health
Sciences Centre, London, UK; Hitesh Shetty, MSC, The National Institute for Health Sciences Centre, London, UK; Hitesh Shetty, MSC, The National Institute for Health
Research (NIHR) Biomedical Research Centre (BRC) at South London and Maudsley NHS Foundation Trust and the Institute of Psychiatry, Psychology \& Neuroscience at King's College London, King's Health Partners Academic Health Sciences Centre, London, UK; Rosalind Ramsay, MBBS, South London and Maudsley NHS Foundation Trust, Bethlem Royal Hospital, Kent, UK; Rocio Perez-Iglesias, PhD, CIBERSAM: Centro Investigación Biomédica en Red Salud Mental, Santander, Spain; Philip McGuire, PhD, King's College London, Department of Psychosis Studies, Institute of Psychiatry, Psychology \& London, Department of Psychosis Studies, Institute of Psychiatry, Psychology \&
Neuroscience, London, UK, and South London and Maudsley NHS Foundation Trust, Bethlem Royal Hospital, Kent, UK

Correspondence: Eugenia Kravariti, Institute of Psychiatry, Psychology and Neuroscience, King's College London, PO Box 63, De Crespigny Park, SE5 8AF, UK. Email: eugenia.kravariti@kcl.ac.uk

First received 11 Jan 2017, final revision 11 Nov 2017, accepted 14 Nov 2017
}

\section{Acknowledgments}

We thank the patients and staff of the Lambeth South Psychosis Recovery and Support Community Service in London, UK, for making this study possible. Special thanks are extended to Mr Garry Moriarty, Clinical Systems Manager of the SLaM Clinical Systems Team, who provided the anonymous data on the number of SMS appointment reminders that were sent without error to the study participants.

\section{Supplementary material}

Supplementary material is available online at https://doi.org/10.1192/bjo.2017.7.

\section{References}

1 NHS England. NHS England Using Technology to Beat Cost of Missed Appointments. NHS England, 2014 (http://www.england.nhs.uk/2014/03/05/ missed-appts/).

2 Mitchell AJ, Selmes T. Why don't patients attend their appointments? Maintaining engagement with psychiatric services. Adv Psychiatr Treat 2007; 13: 423-44.

3 Sims $\mathrm{H}$, Sanghara $\mathrm{H}$, Hayes $\mathrm{D}$, Wandiembe $\mathrm{S}$, Finch $\mathrm{M}$, Jakobsen $\mathrm{H}$, et al. Text message reminders of appointments: a pilot intervention at four community mental health clinics in London. Psychiatr Serv 2012; 63: 161-8.

4 Killaspy H, Banerjee S, King M, Lloyd M. Prospective controlled study of psychiatric out-patient nonattendance: characteristics and outcome. Br J Psychiatry 2000; 176: $160-5$

5 Appleby L, Shaw J, Amos T, McDonnell R, Harris C, McCann K, et al. Suicide within 12 months of contact with mental health services: national clinical survey. BMJ 1999; 318: 1235-9.

6 Hasvold P, Wootton R. Use of telephone and SMS reminders to improve attendance at hospital appointments: a systematic review. J Telemed Telecare 2011; 17: 358-64.

7 Pijnenborg GHM, Withaar FK, Evans JJ, van den Bosch RJ, Brouwer WH. SMS text messages as a prosthetic aid in the cognitive rehabilitation of schizophrenia. Rehabil Psychol 2007; 52: 236-40.

8 Pijnenborg GH, Withaar FK, Brouwer WH, Timmerman ME, van den Bosch RJ, Evans JJ. The efficacy of SMS text messages to compensate for the effects of cognitive impairments in schizophrenia. Br J Clin Psychol 2010; 49: 259-74.

9 Branson CE, Clemmey $\mathrm{P}$, Mukherjee $\mathrm{P}$. Text message reminders to improve outpatient therapy attendance among adolescents: a pilot study. Psychol Serv 2013; 10: 298-303.

10 Berrouiguet S, Baca-Garcia E, Brandt S, Walter M, Courtet P. Fundamentals for future mobile-health (mHealth): a systematic review of mobile phone and webbased text messaging in mental health. J Med Internet Res 2016; 18: e135.

11 Thomas IF, Lawani AO, James BO. Effect of short message service reminders on clinic attendance among outpatients with psychosis at a psychiatric hospital in Nigeria. Psychiatr Serv 2017; 68: 75-80.

12 Jeste DV, Palmer BW, Appelbaum PS, Golshan S, Glorioso D, Dunn LB, et al. A new brief instrument for assessing decisional capacity for clinical research. Arch Gen Psychiatry 2007; 64: 966-74.

13 Perera G, Broadbent M, Callard F, Chang CK, Downs J, Dutta R, et al. Cohort profile of the South London and Maudsley NHS Foundation Trust Biomedical Research Centre (SLaM BRC) Case Register: current status and recent enhancement of an Electronic Mental Health Record-derived data resource. BMJ Open 2016; 6: e008721

14 Bogart K, Wong SK, Lewis C, Akenzua A, Hayes D, Prountzos A, et al. Mobile phone text message reminders of antipsychotic medication: is it time and who should receive them? A cross-sectional trust-wide survey of psychiatric inpatients. BMC Psychiatry 2014; 14: 15.

15 Firth J, Cotter J, Torous J, Bucci S, Firth JA, Yung AR. Mobile phone ownership and endorsement of "mHealth" among people with psychosis: a meta-analysis of cross-sectional studies. Schizophr Bull 2016; 42: 448-55.

16 Phillips Hey S, Kimmelman J. The questionable use of unequal allocation in confirmatory trials. Neurology 2014; 82: 77-9.

17 World Health Organization. ICD-10 Classifications of Mental and Behavioural Disorder: Clinical Descriptions and Diagnostic Guidelines. World Health Organization, 1992. 